\title{
Vantagens da histerectomia vaginal comparado à histerectomia abdominal em mulheres sem prolapso genital
}

\author{
Advantages of vaginal hysterectomy compared to hysterectomy abdominal in women \\ without genital prolapse \\ Ventajas de la histerectomía vaginal comparado a la histerectomía abdominal en mujeres \\ sin prolapso genital
}

Stéfano dos Santos Adorno1*, Juliana Veras de Mesquita², Luís Alberto Leon Camac', Fernanda Goulart Nogueira da Silva², Moisés Castro Saback¹.

\section{RESUMO}

Objetivo: Comparar as vantagens da histerectomia vaginal à histerectomia abdominal em mulheres sem prolapso genital, evidenciando os benefícios do procedimento pela via vaginal. Métodos: Foi realizada uma revisão da literatura em textos de revisão, artigos científicos do tipo de revisão de casos, revisões sistemáticas e estudos controlados no MEDLINE e PubMed (2007-2017), escritos na língua portuguesa, inglesa e espanhola, para a promoção de um estudo básico, exploratório e qualitativo fundamentado. Resultados: Observou-se que dentre as vantagens da histerectomia vaginal à abdominal estão a demanda de menor tempo operatório, ser minimamente invasiva, ter baixa morbidade e sem a existência de cicatriz visível, necessita de menor tempo para o retorno às atividades cotidianas, ter baixa incidência de complicações intra-operatórias, possuir ótima relação custo-benefício, com menor perda sanguínea durante a operação, implicar custos bem mais baixos para o Sistema de Saúde, com menor tempo de deficiência e hospitalização. Conclusão: É preciso disseminar a prática da histerectomia vaginal dando ênfase às cirurgias vaginais nos programas de Residência Médica, associado a treinamentos práticos com supervisão de profissionais experientes. Dessa forma, é possível adquirir vivência e reverter o atual quadro de redução, a cada ano, das histerectomias vaginais.

Palavras-chave: Histerectomia, Vaginal, Abdominal, Prolapso genital.

\begin{abstract}
Objective: To compare advantages of vaginal hysterectomy to abdominal hysterectomy in women without genital prolapse, demonstrating benefits of vaginal procedure. Methods: A review of literature in review texts, scientific articles of type of case review, systematic reviews and controlled studies in MEDLINE and PubMed (2007-2017), written in portuguese, english and spanish languages, were carried out to promote a basic exploratory and qualitative study. Results: It was observed that among advantages of vaginal hysterectomy to abdomen are demand for shorter operative time, to be minimally invasive, to have low morbidity and without the presence of a visible scar, to require less time to return to daily activities, to have low incidence of intraoperative complications, have an excellent cost-benefit ratio, with lower blood loss during the operation, imply much lower costs for the Health System, with less time of disability and hospitalization. Conclusion: It is necessary to disseminate the practice of vaginal hysterectomy by emphasizing vaginal surgeries in Medical Residency programs, associated with practical training supervised by experienced professionals. In this way, it is possible to acquire experience and reverse current reduction in vaginal hysterectomies each year.
\end{abstract}

Key words: Hysterectomy, Vaginal, Abdominal, Genital prolapse.

1 Universidade Estadual do Amazonas (UEA), Manaus - AM. * E-mail: stefanoadorno.med@hotmail.com.

2 Universidade Federal do Amazonas (UFAM), Manaus - AM. 


\section{RESUMEN}

Objetivo: Comparar las ventajas y beneficios de la histerectomía vaginal sobre la histerectomía abdominal en mujeres con ausencia de prolapso genital. Métodos: Fue realizado una revisión de la literatura en textos de revisión, artículos científicos asociados a estudios de casos, estudios sistemáticos y del tipo controlados, extraídos de la base de datos del MEDLINE y PubMed (2007-2017), escritos en lengua portuguesa, inglesa y española, para la promoción de un estudio básico, exploratorio y cualitativo fundamentado. Resultados: Se observó que dentro de las ventajas de la histerectomía vaginal, comparada con la vía abdominal, se encuentra un menor tiempo operatorio, ser una cirugía del tipo mínimamente invasiva con baja morbilidad y ausencia de cicatriz visible; así como, retorno precoz a las actividades cotidianas, baja incidencia de complicaciones intraoperatorias, mejor relación costo- beneficio, menor perdida sanguínea durante el acto operatorio, menores costos para el Sistema de Salud y menor tiempo de estadía hospitalaria. Conclusión: Es necesario incentivar y diseminar la práctica de la histerectomía vaginal, dando énfasis en la formación práctica de los médicos residentes de los programas de Residencia Médica bajo la supervisión de profesionales competentes. De esta manera, será posible adquirir vivencia y revertir el actual cuadro de disminución, que a cada año se viene observando en relación a la práctica de las histerectomías vaginales.

Palabras clave: Histerectomía, Vaginal, Abdominal, Prolapso genital.

\section{INTRODUÇÃO}

Hystera consiste numa palavra de origem grega que significa útero, sendo este um órgão feminino que possui formato de pera, além de ser de fundamental importância para o ciclo reprodutivo humano, uma vez que no útero realiza-se todo o desenvolvimento embrionário (MELO e BARROS, 2009).

Para determinadas culturas e sociedades humanas, cabe depreender que o útero é recorrentemente considerado um símbolo de feminilidade e de sexualidade feminina, mesmo que variados estudos tenham demonstrado que o prazer feminino não é restritivo somente aos órgãos sexuais, envolvendo todo o corpo, de forma que cabe à mulher conhecer todo o seu funcionamento (MELO e BARROS, 2009).

Histerectomia é o ato cirúrgico em que o útero é retirado. Denomina-se de histerectomia abdominal aquela cirurgia pela qual o acesso ao útero é alcançado pela face anterior do abdômen e, por outro lado, a histerectomia vaginal decorre quando o útero é abordado pela vagina (ZINK, 1988).

Complementando entendimentos, destaque ao fato de que a prática de histerectomia se refere a um dos procedimentos cirúrgicos mais frequentes em execução na atualidade, especialmente pelo fato de ser uma intervenção cirúrgica ginecológica indicada para o tratamento de diversas doenças (PIAZZA et al., 2011).

A realização de tal cirurgia não envolve apenas a preocupação da mulher com o ato cirúrgico em si, compreendendo também as suas expectativas no que tange à histerectomia, à vivência pós-operatória, aos motivos, crenças e significados que são recorrente e culturalmente atribuídos ao útero. A referida abrangência se deve, necessariamente, ao fato de que este órgão é considerado expressamente importante para o desempenho do papel da mulher em sociedade, que seria: a maternidade (SALVADOR et al., 2008; SILVA et al., 2010).

Relevante dimensionar que a prática de histerectomia consiste em uma cirurgia irreversível, levando a uma mudança integral da região reprodutiva corporal da mulher, especialmente do útero que é um órgão do corpo feminino impregnado de simbolismos e significados (SILVA et al., 2010), mitos estes que são, geralmente, interpretados no imaginário das mulheres, levando em consideração aquilo que se entendia sobre o significado da mulher ficar sem o útero, ficando inútil aos olhos da sociedade que, tradicionalmente, vê a mulher como elemento de reprodução (VILLAR e SILVA, 2009).

Além disso, há a percepção consagrada de que a histerectomia consiste num procedimento de baixa morbidade, possuindo resultados geralmente confiáveis e sendo, recorrentemente, considerada segura. Importante enfatizar a dimensão do diagnóstico que se coloca como responsável pela realização da grande maioria das histerectomias, tanto no Brasil, quanto no mundo, refere-se ao sangramento uterino secundário a uma condição de leiomiomatose uterina (PIAZZA et al., 2011). 
Dawood et al. (2009) demonstram que as histerectomias abdominais e vaginais compreendem as duas principais modalidades operacionais utilizadas para o tratamento das mais variadas condições e doenças uterinas; entretanto, as indicações para a seleção de um procedimento específico em qualquer que seja a configuração patológica da paciente podem não ser definidas efetivamente.

Com base nos estudos de Wilcox et al. (1994), um dos primeiros desenvolvidos sobre esta técnica, a histerectomia é uma das cirurgias ginecológicas mais realizadas em todo o mundo. Dentre as indicações mais frequentes para a aplicação deste procedimento estão: as doenças benignas, como, por exemplo, a leiomiomatose uterina, a endometriose, as hiperplasias, dentre outras.

Historicamente, Costa et al. (2003) noticia que desde o início do século XIX relatos clínicos evidenciam uma vasta preferência pela técnica vaginal para que possam ser realizados os procedimentos de histerectomia, objetivando principalmente tratar doenças benignas do útero, porém, nesse momento histórico, os resultados eram pouco efetivos e animadores, levando a comunidade médica a buscar por uma nova técnica: a abdominal.

Entende-se que a técnica de histerectomia abdominal surgiu quase que por acaso, especificamente em meados do século XIX, emergindo como um tratamento para úteros leiomiomatosos, porém, mesmo com a nova técnica o desfecho clínico das mulheres com esta patologia específica quase sempre era, em sua totalidade, o óbito, especialmente em função do alto grau de agressão que o procedimento em questão proporcionava (COSTA, 2003).

Porém, seguindo os direcionamentos da literatura científica específica, é indiscutível que a histerectomia vaginal simples é menos invasiva do que o procedimento por via abdominal, uma vez que é dispensável a incisão abdominal, podendo, assim, ser realizada sem a aplicação de anestesia geral e que ainda se caracteriza por possuir um menor tempo cirúrgico, potencialmente minimizando os riscos operatórios (COSTA et al., 2003).

\section{MÉTODOS}

Foi realizada uma revisão da literatura em textos de revisão, artigos científicos do tipo de revisão de casos, revisões sistemáticas e estudos controlados no MEDLINE e PubMed (2000-2017), escrito na língua portuguesa, inglesa e espanhola. Como critérios de exclusão, não foram selecionados textos que não estão relacionados com o tema estudado.

Foram utilizados os seguintes termos (descritores): 1) Histerectomia (hysterectomy, histerectomía); 2) Vaginal (vaginal); 3) Abdominal (abdominal); 4) Prolapso genital (genital prolapse).

\section{RESULTADOS E DISCUSSÃO}

Gómez (2012) evidencia que as principais indicações para a realização de histerectomia vaginal são semelhantes àquelas para histerectomia abdominal. Sendo possível dimensionar que sempre que há uma indicação para histerectomia e contraindicações absolutas não são dimensionadas, a via vaginal pode ser entendida como a melhor escolha em viabilização.

Desta feita, de forma geral, há a observância de que a via vaginal compreende o envolvimento de menos trauma cirúrgico para o paciente, possibilitando recuperação da vida cotidiana e do ritmo intestinal mais rápido, ademais, compreende-se que em mulheres obesas, idosas ou ainda aquelas que apresentem úteros atróficos a abordagem cirúrgica abdominal promove o oferecimento de maiores dificuldades na técnica, independentemente da possibilidade de ser observada maior morbidade do que a prática realizada pelo caminho vaginal.

No que tange aos benefícios, recorrentemente se expressa que a histerectomia vaginal compreende uma via segura e simples, cabendo aos ginecologistas, de forma geral, promoverem estímulos ao desenvolvimento e à prática do procedimento, também denominada de "histerectomia por orifício natural" ou de "histerectomia sem cicatriz visível" (METTLER et al., 2010). 
Sendo assim, o sucesso em meio a realização da histerectomia vaginal é intrinsecamente dependente da indicação e da técnica a serem corretamente aplicadas (METTLER et al., 2010), o procedimento em questão consiste numa cirurgia factível frente a grande maioria dos casos patológicos sem prolapso genital apresentados, nos quais revelam-se baixa frequência de complicações intra e pós-operatórias, além de curto tempo de internação e de grande comorbidade (GOLLOP et al., 2012).

Corroborando com tais percepções e levando em consideração as disposições apresentadas pelos autores Noviello et al. (2003), há o entendimento de que a histerectomia por via vaginal apresenta uma extensa gama de vantagens em relação à promoção da histerectomia por via abdominal, especialmente pelo fato de que a primeira proporciona um menor envolvimento e manipulação das alças intestinais, o tempo de internação da paciente é categoricamente menor, há menos dor no pós-operatório e maior rapidez na recuperação, não deixando cicatriz abdominal, sendo relevante o posicionamento de que a histerectomia vaginal, de forma geral, apresenta custos mais baixos para o Sistema de Saúde como um todo.

Importante evidenciar que a American College of Obstetricians and Gynecologists (2008) define a histerectomia vaginal como sendo o procedimento de extração do útero através da vagina, que possui como vantagens centrais: cicatrizes não visíveis; em comparação com a realização de cirurgia abdominal, apresenta-se como um procedimento mais curto, com período de hospitalização mais breve, retorno mais rápido para a realização de atividades cotidianas, menor recorrência de infecções e menos dor.

Ademais, há a existência de variados estudos que demonstram e destacam as inúmeras vantagens da condução de uma histerectomia vaginal em detrimento da abordagem por via abdominal, dentre as quais destacam-se maior segurança, menores custos, menor morbidade e, ainda, uma melhor tolerância em pacientes que apresentam alto risco cirúrgico ou que encontrem-se em estado menopáusico ou pósmenopáusico (LUCERO, 2010; SALINAS et al., 2006).

E, assim, o Colégio Americano de Obstetras e Ginecologistas produziu um relatório bibliográfico acerca de que o procedimento de histerectomia vaginal está associado expressamente a melhores resultados e menos complicações do que a realização de uma histerectomia abdominal ou laparoscópica, que são mais agressivas e possuem maiores complicações (ACOG, 2008).

Ademais, com base numa revisão da base Cochrane que analisou os resultados de 34 estudos randomizados e controlados de histerectomia vaginal, laparoscópica e abdominal, foi passível concluir que os melhores resultados sob um ponto de vista clínico referem-se àqueles procedimentos promovidos por cirurgia vaginal, além disso, é de relevância pontuar que quando uma histerectomia vaginal não fosse possível, a histerectomia laparoscópica apresenta vantagens comparáveis em relação à histerectomia abdominal, que é caracterizada por sua agressividade (SHETH, 2010).

Em conformidade com os resultados apresentados por Costa et al. (2003), a histerectomia vaginal causou menor nível de perda sanguínea intra-operatória; assim como intensidade reduzida nas dores pósoperatórias, permanência hospitalar diminuída e retorno mais rápido às atividades corriqueiras dos pacientes que passam por este procedimento.

Segundo os Dawood et al. (2009), ainda esclarecendo os benefícios da técnica de histerectomia por via vaginal, observa-se que este procedimento clinicamente está associado a menos condições febris, tempo reduzido (ou nenhum tempo) de infecção da ferida, menor custo econômico e, também, menores situações de sangramento que necessitem de transfusão ou reingressão ao hospital do que a prática de histerectomia abdominal.

Dessa forma, observa-se que principal indicação das operações por via abdominal seriam leiomiomas, por outro lado, a indicação central para a realização de histerectomia vaginal refere-se a mulheres com prolapso uterovaginal (DAWOOD et al., 2009).

Histerectomias realizadas pela via vaginal compreende o oferecimento de menos complicações, estadias hospitalares geralmente mais curtas, além de retorno mais rápido às atividades corriqueiras da paciente. 
Mesmo com tais potencialidades, a abordagem abdominal continua a ser dominante em meio aos prontuários com incidência mundial (DUHAN, 2014).

Para mais, é de importância depreender acerca do fato de que a habilidade e a experiência do cirurgião representam fatores com o desempenho de um papel fundamental em meio aos momentos de determinação de qual a rota de aproximação a ser escolhida quando de uma histerectomia, sendo importante enfatizar que o procedimento vaginal foi convencionado para mulheres com prolapso uterino ou pélvico, porém, pode ser aplicado em pacientes que não apresentam esta condição patológica (DUHAN, 2014).

Apesar da via vaginal ser a preferida para a histerectomia, há menos histerectomias vaginais em comparação com outras vias. Isso é decorrente dos obstáculos à realização de histerectomia vaginal que incluem treinamento limitado, menor número de histerectomias sendo realizadas por cirurgiões individuais e maior diversidade de abordagens cirúrgicas, o que diminui ainda mais o número de cirurgias vaginais realizadas no treinamento e na prática (BERKOWITZ et al., 2018)

Programas de residência em obstetrícia e ginecologia (OB / GYN) iniciaram programas de rastreamento com base na trajetória de carreira esperada de um residente a fim de prever o aumento das histerectomias vaginais $(\mathrm{HV})$ e outros procedimentos limitados realizados por cada residente. Dave et al. analisaram o Relatório Nacional de Dados de Casos OB / GYN e o Relatório de Tendências de Correspondências da Irmandade publicados entre 2010 a 2014. A partir dessa análise, concluíram que esse tipo de rastreamento tem o potencial de aumentar significativamente o treinamento de HV para residentes que vão para campos onde esse procedimento deve ser rotineiramente realizado em mulheres que precisam de uma histerectomia. Vale ressaltar que o número de HV nos EUA é relativamente baixo. Ou seja, o rastreamento pode oferecer uma oportunidade para restaurar especialistas em obstetrícia e ginecologia com a HV.

Embora a utilização do procedimento de histerectomia vaginal sem prolapso ainda não tenha passado por generalização, especialmente no Brasil, existindo estudos que trazem relatos que esta técnica específica possui potenciais vantagens sobre a técnica laparoscópica e a abordagem abdominal, especialmente no que tange ao tempo cirúrgico mais curto, taxas de ocorrências de complicações mais baixas, custos mais baixos especialmente em função de um menor tempo de deficiência e hospitalização (CORREA-OCHOA et al., 2011).

Tal como reportam Freitas et al. (2016), lesões na bexiga, infecções urinárias, lesões retais, sensações de evacuação incompleta, infecção e hematoma de cúpula vaginal, embolia pulmonar, infecção pulmonar, anemia, trombose venosa pélvica, coagulação intravascular disseminada, hemorragia, choque hipovolêmico e sepse são as principais complicações referenciadas no estudo que se associam à realização do procedimento de histerectomia vaginal sem prolapso genital.

De modo complementar, as complicações advindas mais frequentemente da execução da técnica de histerectomia vaginal seriam a lesão vesical, o hematoma de cúpula, infecções e tromboembolismo.

Em conformidade com Manríquez et al. (2013), observam-se evidências suficientes que possibilitam a conclusão que, em meio a grande maioria dos esclarecimentos constantes na literatura científica especializada, a via vaginal compreende a rota ideal para que seja feito um procedimento de histerectomia, em especial, devido ao fato de que tal abordagem é condizente com pequenas complicações - geralmente relacionadas com a presença de comorbidades, especificamente doenças crônicas como hipertensão, diabetes, dentre outras -, recuperação rápida, grande custo-benefício e retorno ágil dos pacientes para a condução de suas atividades rotineiras e de sua vida como um todo.

Importante depreender que os dados expostos pelos autores Manríquez et al. (2013), encontram-se em concordâncias com as referências estatísticas nacionais e internacionais existentes em relação à incidência de complicações intra-operatórias e à frequência de conversão da cirurgia de histerectomia de via vaginal para abdominal, destacando-se a alta porcentagem de histerectomias realizadas com canal vaginal versus 
abdominal no Hospital da Universidade do Chile - local do estudo de Marínquez et al. (2013) -, com a observação de baixa incidência de complicações intra-operatórias.

Os autores Aarts et al. (2015) dão atenção ao fato de que os benefícios e prejuízos dimensionados anteriormente parecem ser, intrinsecamente, dependentes da habilidade cirúrgica, pressuposto este que pode influenciar profundamente a decisão de qual procedimento seguir.

De forma conclusiva, Aarts et al. (2015) depreendem que quando a HV (Histerectomia Vaginal) não é possível, a condução de uma HL (Histerectomia Laparoscópica) pode evitar a necessidade de ser realizada uma HA (Histerectomia Abdominal), porém, é fundamental evidenciar o posicionamento de que HL está recorrentemente associada com a ocorrência de maiores lesões do trato urinário.

Por fim, de forma geral, segue em meio às tabelas abaixo (Quadro 1) os principais benefícios e desvantagens que se envolvem com a execução de histerectomia vaginal e com a execução de histerectomia abdominal em mulheres sem prolapso genital, e assim, têm-se:

Quadro 1. Benefícios e Desvantagens: histerectomia vaginal e histerectomia abdominal.

\begin{tabular}{|c|c|}
\hline \multicolumn{2}{|c|}{ HISTERECTOMIA VAGINAL } \\
\hline Benefícios & Desvantagens \\
\hline 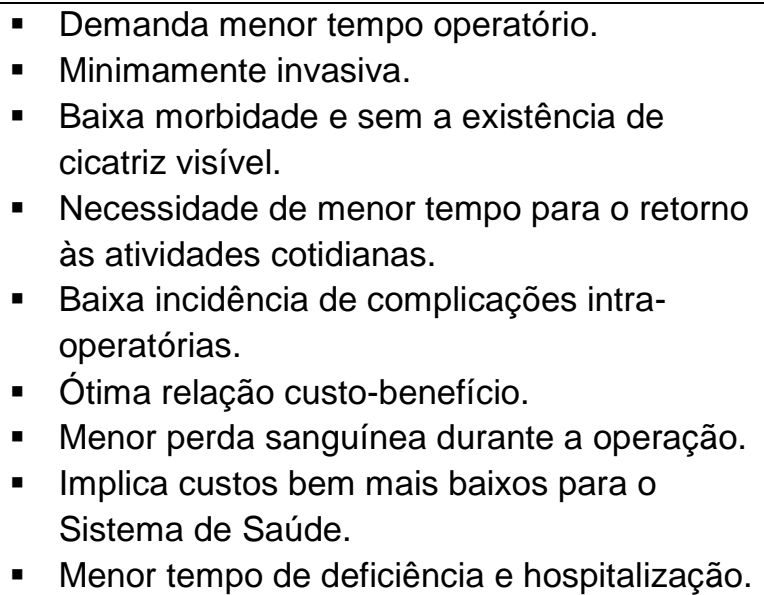 & $\begin{array}{l}\text { - Incisão na vagina. } \\
\text { - Relutância dos ginecologistas em realizar } \\
\text { histerectomias vaginais em pacientes sem } \\
\text { prolapso genital. } \\
\text { - Risco de quadro infeccioso em pacientes com } \\
\text { sobrepeso ou em estado de obesidade. } \\
\text { - Frequência de conversão da cirurgia de } \\
\text { histerectomia de via vaginal para abdominal. } \\
\text { - Possibilidade de lesão vesical, hematoma de } \\
\text { cúpula, infecções e tromboembolismo. } \\
\text { - Recorrente uso da laparoscopia para a cirurgia } \\
\text { vaginal em casos complexos. }\end{array}$ \\
\hline \multicolumn{2}{|c|}{ HISTERECTOMIA ABDOMINAL } \\
\hline Benefícios & Desvantagens \\
\hline $\begin{array}{l}\text { - Menor taxa de reoperação e reincidência. } \\
\text { - Pode ser feita se existirem aderências, se o } \\
\text { - Possibilita uma boa visualização para o } \\
\text { cirurgião. } \\
\text { - Não há nenhuma limitação a respeito do } \\
\text { tamanho do útero. } \\
\text { - Permite uma exploração ampla dos órgãos } \\
\text { - Podominais-pélvicos. } \\
\text { menos experientes. }\end{array}$ & $\begin{array}{l}\text { - Demanda maior tempo operatório. } \\
\text { - Necessidade de maior tempo para o retorno às } \\
\text { - } \text { atividades cotidianas. } \\
\text { - Maior perda sanguínea durante a operação. } \\
\text { - } \text { operatória. } \\
\text { - Aumento da incidência de peritonite, originando } \\
\text { profundas complicações vasculares e } \\
\text { pulmonares. } \\
\text { - Difícil de ser realizada em pacientes obesos. } \\
\text { - A incisão abdominal geralmente encontra-se } \\
\text { associada à morbidade da dor, infecção, } \\
\text { deiscência, evisceração, desconforto ou } \\
\text { - } \text { desenvolvimento de hérnia. } \\
\text { - Possibilidade de infecção do trato urinário. }\end{array}$ \\
\hline Fonte: Autor, 2018. & $\begin{array}{l}\text { Em momento intraoperatório, a paciente pode } \\
\text { sofrer de hemorragia. }\end{array}$ \\
\hline
\end{tabular}




\section{CONCLUSÃO}

Depreende-se, com o presente trabalho, que a abordagem abdominal continua a ser a forma mais comum de histerectomia realizada em todo o mundo, porém, é de entendimento consagrado que o acesso uterino pela via vaginal se associa à presença de menores complicações, estadia hospitalar curta, recuperação rápida e, para o Sistema de Saúde, custos mais baixos. Portanto, como enfatizado pelos estudos de Costa et al. (2003), é fundamental, no sentido de disseminar a prática da histerectomia vaginal, dar ênfase às cirurgias vaginais nos programas de Residência Médica, com treinamentos práticos supervisionados por profissionais experientes. Só assim que se adquire vivência e reverte a atual situação de redução, a cada ano, de histerectomias por via vaginal. Além disso, faz-se necessária associar os treinamentos práticos com a reciclagem constante dos cirurgiões em atividade, possibilitando a atualização das técnicas e, consequentemente, dos resultados e desempenho.

\section{REFERÊNCIAS}

1. AARTS JWM, NIEBOER TE, JOHNSON N, TAVENDER E, GARRY R, MOL BWJ, KLUIVERS KB et al. Surgical approach to hysterectomy for benign gynaecological disease. Cochrane Database of Systematic Review 2015, Issue 8. Art. No.: CD003677.

2. ACOG - The American College of Obstetricians and Gynecologists. La histerectomía. Procedimientos Especiales, 2008.

3. BERKOWITZ LR, FOUST-WRIGHT CE. Vaginal hysterectomy. Uptodate. Literature review: Sep 2018. Consulted October 2018.

4. CORREA-OCHOA JL, TIRADO-MEJÍA JA, MEJÍA-ZÚÑIGA JF, TIRADO-HERNÁNDEZ M, GÓMEZ-IBARRA EA, ARANGO AM et al. Histerectomía vaginal sin prolapso: estudio de cohorte. Medellín (Colombia) 2008- 2010. Rev colomb obstet ginecol [Internet]. 2011;62(1):45-50.

5. COSTA AAR, AMORIM MR, CURSINO T et al. Histerectomia Vaginal versus Histerectomia Abdominal em Mulheres sem Prolapso Genital, em Maternidade-Escola do Recife: Ensaio Clínico Randomizado. RBGO 2003, 25(3):169-176.

6. DAWOOD NS, MAHMOOD R, HASEEB $N$ et al. Comparison of vaginal and abdominal hysterectomy: peri- and post-operative outcome. J Ayub Med Coll Abbottabad 2009;21(4):116-120.

7. DUHAN N. Techniques of Hysterectomy. Department of Obstetrics and Gynecology. Sharma Post Graduate Institute of Medical Sciences. India: ROHTAK, 2014.

8. FREITAS CB, GOMES NP, CAMPOS LM, ESTRELA FM, CORDEIRO KCC, SANTOS RM et al. Complicações pós-cirúrgicas da histerectomia: revisão integrativa. Revista Baiana de Enfermagem, Salvador, abr./jun. 2016;30(2):1-11.

9. GOLLOP TR, SANTOS AG, ROSSI AGZ, BIANCHI RF et al. Histerectomia vaginal em útero sem prolapso - experiência de 6 anos. Einstein. 2012;10(4):462-5.

10. GÓMEZ EP. Histerectomía vaginal. In: Servicio de Obstetricia y Ginecología Hospital Universitario Virgen de las Nieves. Granada, 2012.

11. LUCERO M, SHAH AD. Vaginal hysterectomy for the prolapsed uterus. Clin Obstet Gynecol 2010;53:26-39.

12. MARÍNQUEZ VG, NASER MN, GÓMEZ ML et al. Complicaciones intraoperatorias de la histerectomía vaginal por causas benignas. Experiencia de seis años, Hospital Clínico de la Universidad de Chile. Rev Chil Obstet Ginecol 2013;78(6):432-435.

13. MELO MCB, BARROS EN. Histerectomia e Simbolismo do útero: possíveis repercussões na sexualidade feminina. Rio de Janeiro, Revista Sociedade Brasileira de Psicologia Hospitalar; Dez. 2009;12(2).

14. METTLER L, REICH H, FENG L, PUNTAMBEKAR S, GALLINAT A, STARK $M$ et al. Hysterectomy: current methods and alternatives [editorial]. Obstetrics e Gynecoly International. 2010;20(10):705073.

15. NOVIELLO MB. Prolapso de tuba uterina após histerectomia vaginal: relato de caso. Rio de Janeiro, Revista Brasileira de Ginecologia e Obstetrícia. Nov./Dez. 2003;25(10).

16. PIAZZA MJ, PEIXOTO AP, PEIXOTO RN, URBANETZ AA et al. Histerectomia total versus histerectomia supracervical. FEMINA. Out. 2010;39(10):479-484.

17. SALINAS H, PASTÉN J, NARANJO B, CARMONA S, RETAMALES B, DIAZ G, FRANULIC L et al. Análisis clínico y económico de la histerectomía abdominal versus la histerectomia vaginal en el Hospital Clínico de la Universidad de Chile. Revisión de 2338 casos. Rev Chil Obstet Ginecol 2006;71:227-33.

18. SALVADOR RT, VARGENS OMC, PROGIANTI JM et al. Sexualidade e histerectomia: mitos e realidade. Rev Gaúcha Enferm. 2008 Jun; 29(2):320-3.

19. SHETH SS. Vaginal hysterectomy as primary route for morbidly obese women. Acta Obstet Gynecol 2010;89:971-4.

20. SILVA CMC, SANTOS IMM, VARGENS OMC et al. A Repercussão da histerectomia na vida de mulheres em idade reprodutiva. Esc Anna Nery Rev Enferm. 2010 Jan-Mar; 14(1):76-82.

21. VILLAR ASE, SILVA LR. O Sentimento de Mulheres Submetidas à Histerectomia e a Interferência na Vida Sexual. Revista de Pesq. Cuidad Fundam. Out. 2009;1(2):235-244.

22. WILCOX LS, KOONIN LM, POKRAS R, STRAUSS LT, XIA Z, PETERSON HB et al. Hysterectomy in the United States, 1988-1990. Obstet Gynecol 1994; 83:549-55.

23. ZINK HC. Diccionario Pschyrembel de ginecología y obstetricia. New York: de Gruyter, 1988. 